\title{
Multi Agent System-Based on Case Based Reasoning for Cloud Computing System
}

\author{
Amir Mohamed Talib ${ }^{1}$ and Nour Eldin Mohamed Elshaiekh ${ }^{2}$ \\ ${ }^{1}$ Faculty of Computer and Information Science, Information Technology Department, Al-Imam Muhammad Bin \\ Saud Islamic University, Riyadh, Saudi Arabia \\ ${ }^{2}$ Faculty of Computer Science, Software Engineering Department, Future University, Sudan
}

\begin{abstract}
Cloud Computing is a new technology which use the Internet and central remote servers in order to maintain data and applications. The aim of this paper is to describe about Case Based Reasoning (CBR) which is based on Multi Agent System (MAS) and the implementation in Cloud Computing Environment to assist the Cloud Service Provider (CSP) to deliver a number of services to the cloud users according to their needs. The Introduction Section gives an introduction about Cloud Computing, MAS and CBR Integration, and defines the characteristics and process cycle of CBR. The Second Section describes about the MAS-Based Cloud Computing. The Third Section describes about the CBR in Cloud Computing. The Fourth Section describes the CBR (MAS-Based) for Cloud Computing and Interaction between CBR Agents and components of Cloud Computing. The Final section gives a conclusion about the paper.
\end{abstract}

Keywords: Cloud computing, cloud data storage, cloud service provider, cbr and multi agent system.

\section{Introduction}

\subsection{Cloud Computing}

Cloud computing is a new technology which use the Internet and central remote servers in order to maintain data and applications. The name of "cloud computing" was inspired by the cloud symbol that is often used to represent the Internet in diagrams and flow charts [1]. Cloud computing allows users and businesses to use cloud application without installing any software. They will be able to access their personal files, data and application at any computer as long as there is Internet access [2]. A simple example of cloud computing is Gmail and Yahoo Email. The cloud user does not need to have the software or a server to use them. They only need Internet connection and can start sending email [3].

Cloud computing integrates and provides different types of services such as Data-as-a-Service (DaaS), which allows cloud users to store their data at remote disks and access them anytime from any place. Software-as-a-Service (SaaS), the software and its applications are delivered as services over the Internet. Communication-as-a-Service (CaaS), communication becomes a vital component of the cloud infrastructure due to the needs for a guaranteed Quality of Service (QoS) for network communication grows for cloud systems. Platform-as-a-Service (PaaS), systems software made available over the Internet and Infrastructure-as-a-Service (IaaS), when the hardware made available for cloud users $[4,5,6]$. The foundations of cloud infrastructure provide more flexibility and dynamism in the computing infrastructure than previous forms of distributed systems. The requirements and demands from users for cloud services vary, resulting in complex design and deployment of resources.

\subsection{Case Based Reasoning}

Case-Based Reasoning (CBR) is a method for solving problems by comparing a problem situation to previously experienced ones. The aim is to store information about earlier situations, and when new ones arrive, find the situation that is most similar, and reuse it to match the new problem if the most similar problem does not match sufficiently [7]. This may involve using background knowledge or asking a user. Information about the problem solving experience is learned by the CBR system and the aim is to be able to handle an increased number of situations and also reason more on each situation to certify that it is

*Corresponding author: Address: Address: Faculty of Computer and Information Science, Al-Imam Muhammad Bin Saud Islamic University, Riyadh, Saudi Arabia. E-mail address: ganawa53@yahoo.com 
handled correctly. Each case is a set of features, or attribute-value pairs, that encode the context in which the ambiguity was encountered [8]. The case retrieval algorithm is mostly a simple k-nearest neighbor's algorithm. The basic case-based learning algorithm performs poorly when cases contain many irrelevant attributes.
Unfortunately, deciding which features are important for a particular learning task is difficult. At the highest level of generality, a general CBR cycle may be described by the following four processes as shown in Figure 1:

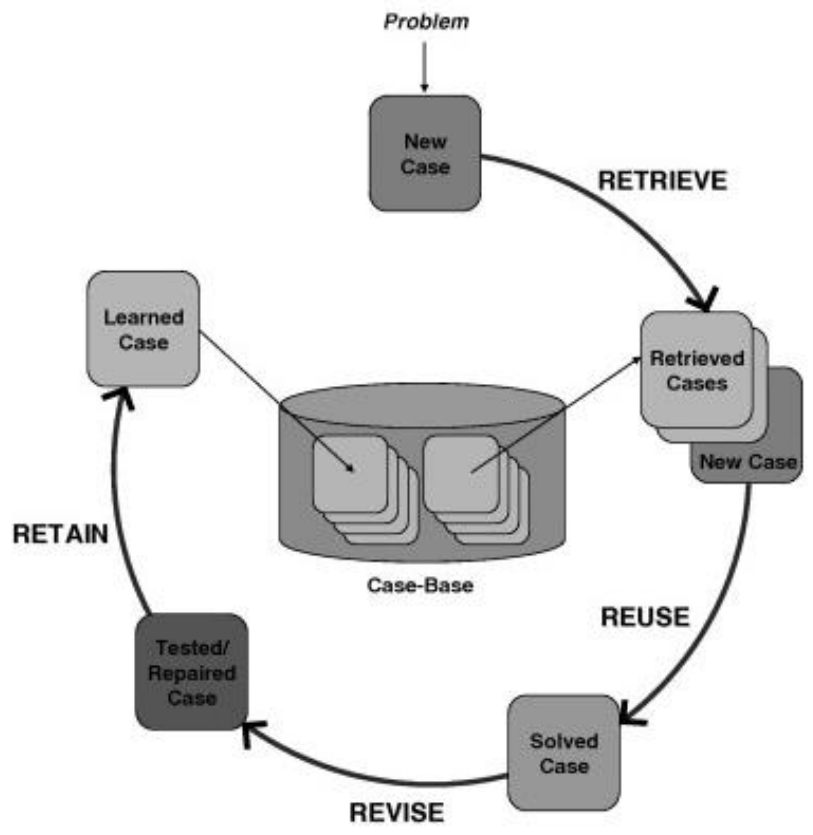

Figure 1. Processes of CBR

CBR methods can be divided into four steps, retrieve find the best matching of a previous case, reuse - find what can be reused from old cases, revise - check if the proposed solution is correct, and retain - learn from the problem solving experience [7].

\section{1) Retrieve}

Retrieving a case means to start with a (partial) new case, and retrieve the best matching previous case. It involves the following subtasks:

Identify features - this may simply be to notice the feature values for a case. This can be filtering out noisy problem descriptors, infer other relevant problem features, check whether the feature values make sense in the given context, or generate expectations of other feature values.

Initially match - usually done in two parts, first an initial matching process which gives a list of possible candidates, which are then further examined to select the best. There are three ways of retrieving a case or a set of cases: By following direct index pointers from the problem features, by searching an index structure, or by searching in a model of domain knowledge.

Select - select a best match from the cases returned by the initially match. The reasoner tries to explain away non-identical features. If the match is not good enough, a better one is sought by using links to closely related cases. The selection process can generate consequences and expectations from each retrieved case, by using an internal model or by asking the user.

\section{2) Reuse}

The focus of reuse is to find the difference between the new and the old case, and find what part of the old case that can be used in the new case. It either involves copying the old solution or adapting it:

Copy - in simple classification, the differences between the old and new case are abstracted away, and the solution is simply copied from the old case.

Adapt - either the solution itself can be (transformed 
and) reused, or the past method that produced the solution can be used.

\section{3) Revise \\ If the solution generated by the last phase is not correct, the system can learn from its failures. This involves:}

Evaluate - try the solution proposed by the reuse-phase in the real environment, and evaluate it.

Repair fault - If the solution evaluated badly, find the errors or flaws of the solution, and generate explanations for them.

\section{4) Retain}

Incorporate what is useful to learn from the problem solving experience into the existing knowledge.

Sub processes are:

Extract - if the problem was solved using an old case, the system can build a new case, or generalize an old case to include the new case as well. If the user was asked, a new case should be constructed. Explanations may be included in the case.

Index - decide what types of indexes to use for future retrieval.

Integrate - modify the indexing of existing cases after the experience, strengthen the weight of features that were relevant, and decrease the weight of features that lead to retrieval of irrelevant cases [7].

\subsection{Multi Agent System and Case Based Reasoning Integration}

Both methods are used for to assist the CSP to deliver a number of services to the cloud users according to their needs and organize and process knowledge to make it available for improving the quality of cloud computing system.

CBR relies heavily on the quality of the data collected, the amount of data, the amount of background knowledge and a way of comparing cases to decide which is most similar. The method is best suited for domains that change, and where we have little knowledge of underlying processes that govern the domain [8].

MASs are ways of extracting information from cloud data storage and can thus be used for extracting information which is relevant for a problem situation. It could also be used to find "unexperienced" problem situations from cloud data storage and represent it as a case, possibly by interacting with a cloud user.

\section{Multi Agent System}

The term "agent" is very broad and has different meanings to different researchers $[9,10]$. One author [9] says that software agents are application programs that communicate with each other in an expressive Agent Communication Language (ACL). Though at first this definition may seem a little simplistic, it allows researchers to clearly identify what constitutes MAS, i.e. agents are just pieces of autonomous code, able to communicate with each other using an ACL. Thus, the assumed view is exactly the same as that presented in [9], but in addition, the agents have unique names as a means of identification.

Agents are considered to be autonomous entities, such as software programs or robots. Their interactions can be either cooperative or selfish. That is, the agents can share a common goal, or they can pursue their own interests. Security plays an important role in the development of MASs and is considered as one of the main issues to be dealt for agent technology to be widely used outside the research community [10].

\section{Multi Agent System-Based Cloud Computing}

In cloud computing, agents offer the flexibility to integrate many different categories of processing within a single cloud computing system. Agent definitions range from descriptions based on a functional analysis of how agents are used in technology to far more ranging expositions based on different interpretations of the role and objectives of artificial intelligence and cognitive science. Artificial intelligence is a very diverse field and agents are used as metaphors for work in many areas.

MAS consist of a number of agents interacting with each other through the Agent Communication Agent (ACL). Agents must be able to interact to achieve goals [11]. Agents may exhibit different types of behaviors when interact with each others such as selfish or benevolent behavior. In CDS scenarios, selfish agents ask for help from other agents if they are overloaded and never offer help such as the agent that serving VIP cloud users for Cloud Service Provider (CSP) service never help other agents for the same 
service. Benevolent agents always provide help to other agents because they consider system benefit is the priority such as the agent that serving normal cloud users for CSP service are always ready to help other agents to complete their tasks $[12,13]$.

\section{CBR based Cloud Computing System}

CBR is concerned with the solution of problems by identifying and adapting similar problems stored in a library of past experience/solutions. In our approach,
CBR techniques are encapsulated into a MAS, which dynamically helping CSPs to deliver a number of services to the cloud users according to their needs. In cloud computing, the CBR agent helps in gaining alternative perspectives on the problem at hand by intervening autonomously during a decision been made by CSP and exposing them interactively to how problems are different, but relevant contexts have been approached and solved.

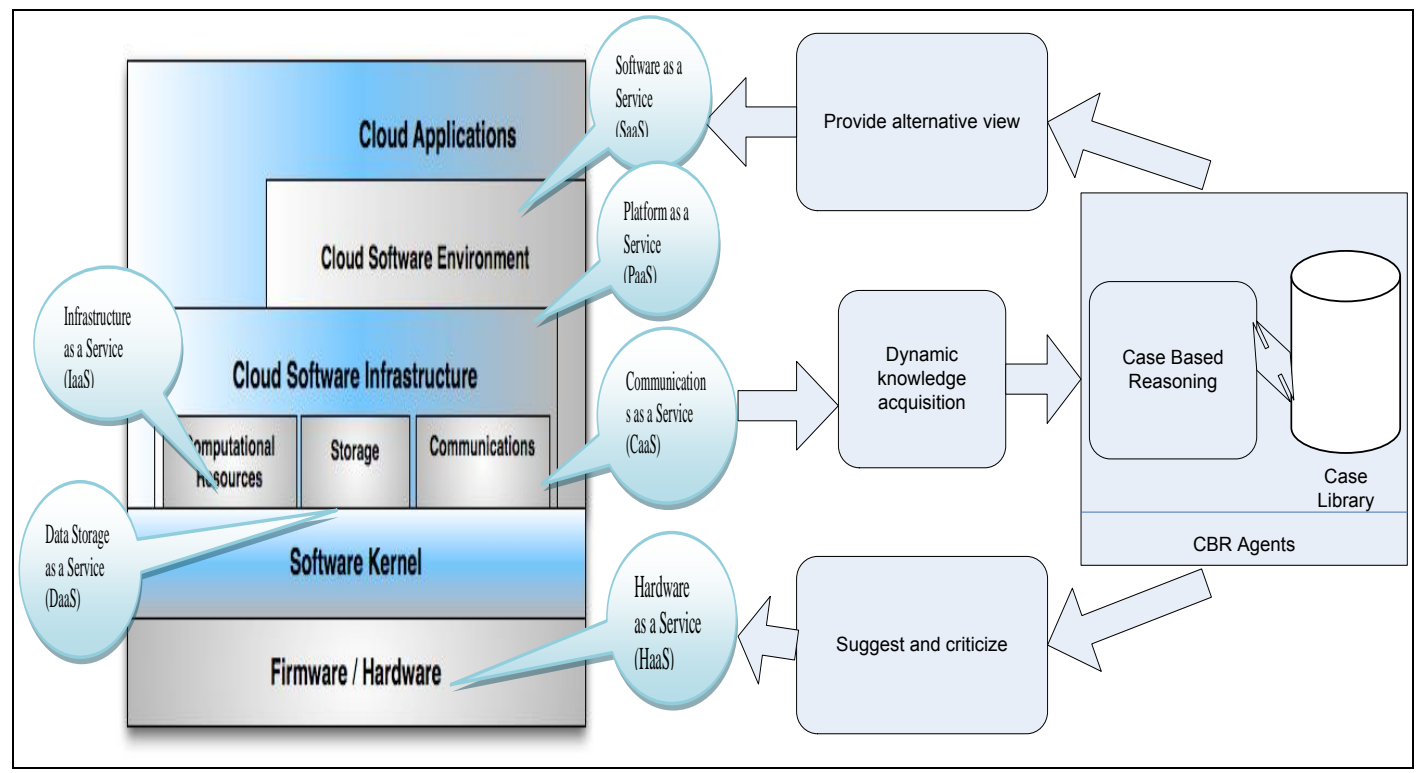

Figure 1. Impact of CBR Agents in Cloud Computing Environment

\section{MAS based CBR and Cloud Computing Components Integration}

The CDS is the main part of cloud computing in which all the services will be delivered to the cloud users through it and has as role to manage all the services. The environment includes the CSP, cloud user and the service. Cloud storage is composed of thousands of storage devices clustered by network, distributed file systems and other storage middleware to provide cloud storage service for users. The typical structure of cloud storage includes storage resource pool, distributed file system, service level agreements (SLA), and service interfaces, etc. Globally, they can be divided by physical and logical functions, boundaries and relationships to provide more compatibilities and interactions. Cloud storage is tending to combine with cloud security, which will provide a more robust security [14].

\section{Conclusions}

The marriage between Clouds and MAS-Based CBR can be convenient for both parties. We discussed in the paper how this can be done and which scientific areas such as cloud computing and issues must be involved to carry out research work that will produce intelligent Cloud services that could be assist the CSPs in delivering the cloud services to the requested cloud users and high-performance MASs on Clouds. The convergence of interests between MASs that need reliable distributed infrastructures and Cloud computing systems that need intelligent software with dynamic, flexible, and autonomous behavior will result in new systems and applications. Both research communities must be aware of this opportunity and should put in place the joint research activities needed to reach that goal. 


\section{References}

[1] Talib, A.M. Atan, R. Abdullah, R., and Azrifah, M. "Towards a Comprehensive Security Framework of Cloud Data Storage Based on Multi Agent System Architecture," Journal of Information Security, Vol. 3, No. 4, 2012, pp. 295-306.

[2] Talib, A.M. Atan, R. Abdullah, R., and Azrifah, M. "CloudZone: Towards an Integrity Layer of Cloud Data Storage Based on Multi Agent System Architecture," Proceeding of the International Conference on Open Systems (ICOS), IEEE, 2011, pp. 127-132.

[3] Neto, P. "Demystifying Cloud Computing," Proceeding of Doctoral Symposium on Informatics Engineering, 2011.

[4] Yang, J., and Chen, Z. "Cloud Computing Research and Security Issues," IEEE, Computational Intelligence and Software Engineering (CiSE), 2010.

[5] Talib, A.M. Atan, R. Abdullah, R., and Azrifah, M. "Security Framework of Cloud Data Storage Based on Multi Agent System Architecture-A Pilot Study," Proceeding of the International Conference on Information Retrieval \& Knowledge Management (CAMP), IEEE, 2012, pp. 54-59

[6] Abdullah, R. Eri, Z.D., and Talib, A.M. "A Model of Knowledge Management System for Facilitating Knowledge as a Service (KaaS) in Cloud Computing Environment," Proceeding of the International Conference on Research and Innovation in Information Systems (ICRIIS), IEEE, 2011, pp. 1-4. [7] Nagaiah, M.D. “Agent-Based CBR for Decision Support System,” 2011.
[8] Althoff, K.-D. "Case-Based Reasoning," Handbook on Software Engineering and Knowledge Engineering, Vol. 1, 2001, pp. 549-587.

[9] Genesereth, M.R., and Ketchpel, S.P. "Software Agents," Commun. ACM, Vol. 37, No. 7, 1994, pp. 48-53, 147.

[10] Mouratidis, H. Giorgini, P., and Manson, G. "Modelling Secure Multiagent Systems," Proceeding of the Second International Joint Conference on Autonomous Agents and Multiagent Systems, ACM, 2003, pp. 859-866.

[11] hen, B. Cheng, H.H., and Palen, J. "Integrating Mobile Agent Technology with Multi-Agent Systems for Distributed Traffic Detection and Management Systems," Transportation Research Part C: Emerging Technologies, Vol. 17, No. 1, 2009, pp. 1-10.

[12] Talib, A.M. Atan, R. Abdullah, R., and Azrifah, M. "Towards New Data Access Control Technique Based on Multi Agent System Architecture for Cloud Computing," Digital Information Processing and Communications, Springer, 2011, pp. 268-279.

[13] Talib, A.M. Atan, R. Abdullah, R., and Azrifah, M. "Ensuring Security and Availability of Cloud Data Storage Using Multi Agent System Architecture," Knowledge Technology, 2012, pp. 343-347.

[14] Zeng, W. Zhao, Y. Ou, K., and Song, W. "Research on Cloud storage Architecture and Key Technologies," Proceedings of the 2nd International Conference on Interaction Sciences: Information Technology, Culture and Human, ACM, 2009, pp. 1044-1048. 